\title{
Mecanismos pessoais e coletivos de proteção e promoção da qualidade de vida para a infância e adolescência
}

\author{
Personal and collective mechanisms for protecting \\ and enhancing the quality of life during childhood and adolescence
}

Maria Conceição O. Costa ${ }^{1}$

Marc Bigras ${ }^{2}$

\footnotetext{
${ }^{1}$ Programa de Pós Graduação em Saúde Coletiva, Núcleo de Estudos e Pesquisas na Infância e Adolescência, UEFS. BR $116, \mathrm{Km} 3$, Campus Universitário, M ódulo VI, Departamento de Saúde. Feira de Santana BA. costamco@hotmail.com 2 Université du Québec à Montréal, UQAM.
}

Abstract Based on recent literature, this paper presents individual and collective strategies for protecting and promoting the quality of life during childhood and adolescence. Addressing the core principles of an all-round protection policy aligned with the U niversal Declaration of Human Rights and the Children's and Adolescents' Statute, it also encompasses practical aspects of professional approaches to children and adolescents in terms of ethicsand confidentiality. Additionally, it discusses the determining factors that shapethe development of individual and collective resilience, on scientific and theoretical bases, in addition to risk factors, health protection and the cognitive and psychosocial development of this segment of the population. The roles played by adults are stressed as protection factors in the macro and micro environments, especially the roles assigned to family members and other adults in the social surroundings such as healthcare practitioners those engaged with education, social welfare and mental health. The important role played by Social Networks is stressed, as a key strategy for implementing interdisciplinary and trans-sector practices designed to enhance well-being and quality of life throughout the entire community.

Key words Health, Quality of life, Childhood, Adolescence
Resumo Neste artigo, são apresentadas, à luz da literatura recente, as estratégias individuais e coletivas voltadas à proteção e promoção da qualidade de vida para a infância e adolescência. São abordados os princípios básicos da política de proteção integral, em acordo com a Declaração U niversal dosD ireitosH umanoseEstatuto da Criança e Adolescente; aspectos práticos da abordagem profissional da criança e do adolescente, quanto à ética econfidencialidade; assim como édiscutido, com bases científicas e teóricas, os determinantes do desenvolvimento da resiliência individual e coletiva, fatores de risco e de proteção à saúde e desenvolvimento cognitivo epsicossocial dessegrupo populacional. Foi enfatizado o papel dosadultos, como fator de proteção, no macro e micro ambientes, especialmente o papel dos familiares, assim como dos adultos do ambiente social como profisssionais da saúde, educação, serviço social, saúdemental. Ressalta-seassim o importantepapel das Redes Sociais, estratégia fundamental de implementação de práticas interdisciplinares e transetoriais com vistas ao bem- estar e qualidade devida para toda a comunidade.

Palavras-chave Saúde, Qualidade de vida, Infância, Adolescência 


\section{Introdução}

A proteção e a promoção da qualidade de vida de crianças e adolescentes representam desafios, cuja amplitude e complexidade ultrapassam aqueles que as agências de saúde pública habitualmente solucionam. Este importante segmento da população é mais vulnerável porque é formado de indivíduos ainda imaturos para enfrentar sozinhos as exigências do ambiente.

Assim como os adultos, crianças e adolescentes têm necessidades de saúde variável, a depender da qualidade de interação entre as esferas biológica, psicológica esocial, de acordo com a etapa de desenvolvimento. Ademais, nós sabemos que, apesar de toda a resiliência de que as crianças são capazes, o comprometimento do seu desenvolvimento normal acarreta maiores riscos de problemas de saúde, os quais podem ser irreversíveis, contrário aos adultos que já encontram-se constituídos. Em suma, as realidades especificas que vivem a infância e adolescência apontam que os esforços voltados à saúde pública necessitam ser mais eficientes, abrangentes e criativos.

A maioria das ações de saúde pública envolvem práticas voltadas diretamente aos adultos, com o objetivo de que estes adotem comportamentos saudáveis, em benefício próprio. Entretanto, as ações de saúde publica voltadas à infância e juventude devem ser indiretas, necessariamente dirigidas por adultos capazes de proteger e cuidar, não somente por uma questão legal ou ética, mas sobretudo por uma questão de garantir a eficácia eo impacto da ação-interven ção. Isto implica que as adequadas intervenções em saúde pública devem, inicialmente, convencer os adultos a adotar comportamentos altruístas em relação à saúde, atitude esta que ben eficiará não somenteas crianças da família, como também aquelas da comunidade. Essas ações apresentam algumas dificuldades na implementação, especialmente em famílias e comunidades desfavorecidas e onde a empatia e a sensibilidade do adulto para a criança encontram-se comprometidas.

Além das dificuldades inerentes à implementação de ações voltadas à promoção e proteção da saúde de crianças e adolescentes (ação indireta), freqüentemente, as intervenções de saúde pública se prestam a compensar as incapacidades do meio ou até mesmo a falta de comprometimento dos adultos. M uitas vezes, essas ações são ignoradas emesmo rejeitadas, por conta da falta decompreensão, responsabilidade ou esclarecimento destes sobre o seu papel na formação e desenvolvimento de crianças e adolescentes. Consideran- do que, neste contexto, o direito da criança pode causar um conflito, a intervenção de saúde pública corre o risco de fazer parte de uma controvérsia, o que pode comprometer a inclusão dos adultos, no processo de proteção à saúde. Felizmente, novos conhecimentos, ferramentas e experiências são disponíveis, graças à mobilização daqueles que trabal ham na procura de melhorar os mecanismos de proteção, cuidados com a saúde eintegração social desse segmento populacional.

Nas próximas páginas, nós veremos que ferramentas, como princípios e legislações que definem os direitos, assim como os conhecimentos que orientam o trabalho de reconciliação das necessidades de adultos e crianças, nos autorizam a pensar e esperar por melhores condições de vida e bem-estar para todos.

\section{Princípios da proteção integral para a infância e adolescência}

A proteção e a qualidade de vida para a infância e a adolescência integram os princípios fundamentais (de atenção e de direitos), que se encontram legitimados em documentos históricos, mundialmente consagrados - a Declaração Universal dos Direitos da Criança (1959) e a Convenção Internacional de Direitos da Criança e Adolescente ${ }^{1,2}$.

No Brasil, os direitos fundamentais à infância e adolescência encontram-se assegurados no Estatuto da Criança e do Adolescente - ECA (1990) que constitui uma avançada legislação, especialmente no que diz respeito à substituição do Código de M enores, cujos princí pios contemplavam medidas de assistência e proteção para os meninos em situação irregular, abandonados, considerados "menores em risco" 3 .

Segundo os princípios da proteção integral, crianças eadolescentes devem ser tratados como sujeitos de direitos (cidadãos) e como grupo prioritário, tendo em vista o estágio de desenvolvimento biopsicossocial, cujas peculiaridades Ihes atribuem necessidades especiais e imediatas, de acordo com a dinâmica do processo de maturacional ${ }^{4}$. Em acordo com esses princípios, profissionais dos diferentes domínios do conhecimento, como exemplo, na área de saúde, exercem um papel prioritário. Os problemas econômicos e políticos que dificultam e às vezes inviabilizam diferentes iniciativas, individuais e degrupos, não devem desmobilizar essas potencialidades, que representam possibilidades de colocar em prática princípios legalmente assegurados, assim como 
cobrar do poder público o cumprimento de políticas contextualizadas, que favoreçam, efetivamente, um ambiente saudável para o desenvolvimento, a integração social (familiar e comunitária) e as condições de vida ${ }^{5,6}$.

Entre os indicadores de desenvolvimento e condições de vida para a infância e adolescência, destacam-se o Índice de Desenvolvimento Infantil - IDI (UNICEF) eo Í ndice de Desenvolvimento Juvenil - IDJ. O IDI é composto pela síntese da qualidade de vida de 0 a 6 anos, obtido pela combinação de cinco indicadores: proporção de crianças com mães de escolaridade precária; com pai de escolaridade precária; cobertura de vacinação (sarampo e DPT); proporção de gestantes com cobertura pré-natal eescolarização brutana pré-escola e creche ${ }^{7}$

À semelhança do índice de Desenvolvimento Humano - IDH (Nações Unidas), o IDJ (2004) foi um índice proposto para a faixa de 15 a 24 anos, que trata das condições de vida da juventude no Brasil, composto pelos indicadores de educação (analfabetismo, escolarização adequada e qualidade do ensino); de saúde (mortalidade por causas internas e causas violentas) e de renda per capita da família ${ }^{8,9}$.

A seguir, estaremos abordando al guns aspectos teórico-conceituais relacionados à temática em questão, os quais integram, efetivamente, 0 contexto das ações e intervenções voltadas à meIhorar osíndices IDI eIDJ, como os aspectos éticos e confidenciais da atenção à infância e adolescência; as condições favoráveis (fatores de proteção) ou desfavoráveis (risco) relacionadas à saúde; o papel dos adultos de referência na formação e no desenvolvimento de crianças e adolescentes e o papel das redes sociais na promoção e proteção da qualidade de vida para a infância e adolescência.

\section{Abordagem dos aspectos éticos eda confidencialidade}

A abordagem profissional de crianças e adolescentes leva em conta os princípios norteadores da bioética, considerando aspectos relacionados ao consentimento legal do atendimento ou intervenção, o sigilo e a confidencialidade, tendo em vista resguardar princípios profissionais básicos quefazem a diferença na qual idade do atendimento, na adesão, assim como no resultado da ação ou intervenção.

0 "Termo de Consentimento Livree Esclarecido" ${ }^{10}$ tem sido 0 instrumento utilizado para a realização de pesquisas com humanos, assim como no atendimento profissional, diante das diferentes ações e intervenções. 0 consentimento livre e esclarecido é definido como "o consentimento do indivíduo, autorização voluntária da participação" que, no caso de crianças e adolescentes, é assinado pelos pais, responsáveis, tendo em vista que, segundo esta resolução, esse grupo populacional, comparado aos adultos, ainda não possui capacidade adequada de discernimento, precisando apenas ser informados ${ }^{11}$.

Esta normativa tem sido amplamente discutida pelos profissionais e grupos que lidam com os direitos humanos, voltados à infância eadolescência, considerando a importância do próprio indivíduo manifestar sua vontade. A resolução 41/ 1995, do Conselho Nacional de Direitos da Criança e Adolescente (M inistério da Justiça); a Resolução 251/97 do Conselho Nacional de Saúdee a Resolução 16/00 do Conselho Federal de Psicologia afirmam a importância dos próprios sujeitos serem informados, manifestarem e participarem efetivamente dos procedimentos e objetivos da interven ção, juntamente com os parentes eresponsáveis. M uito embora os códigos e leis transfiram para as famílias o poder de decisão sobre crianças eadolescentes, a atuação profissional tem sido consensual quanto à importância da participação desses sujeitos, diante do "Termo de Consentimento Livre e Esclarecido", considerando a importância do indivíduo em desenvolvimento compreender e participar efetivamente de decisões relacionadas a si próprio ${ }^{3,12}$.

N os casos de crianças e adolescentes cujas famílias nem sempre cumprem a função protetora, caracterizando situações de risco (situação de rua, vítimas de negligência, abusos físico e sexual, outras circunstâncias), nos casos de ausência da família ou constatado a conivência com a situação de risco vivida pela criança ou adolescente, a Resolução 16/00 do Conselho Federal de Psicologia afirma que o Consentimento Livre e Esclarecido deveser assinado por instituições que prestem assistência legal à infância, como osConselhos Tutelares. Da mesma forma, esses sujeitos devem ser informados sobre objetivos e procedimentos a ser realizados, assim como manifestarem sua vontade ${ }^{12}$.

0 sigilo no atendimento encontra-se pautado nos códigos de ética profissional que, no Brasil, são respaldados pelo Estatuto da Criança e do Adolescente (lei 8069/1990), constituindo um direito do cliente ao sigilo profissional sobre as circunstâncias do atendimento ${ }^{13}$.

Entre os aspectos relacionados ao sigilo na 
abordagem, destaca-se o papel da confidencialidade, que constitui um acordo entre o profissional e o cliente, o pesquisador e o sujeito da pesquisa, o qual permite que seja estabelecida uma relação de confiança mútua. Esta estratégiaénorteada pelos princípios da bioética e constitui um importante apoio profissional à resolução de diferentes problemáticas. A quebra da confidencialidade é admitida quando existir alta probabilidade de um dano (suicídio, homicídio, doenças graves, depressão, outras); um benefício resultante da quebra do acordo, como último recurso ${ }^{4,15}$.

A confidencialidade pode levar a alguns conflitos, diante da possibilidade e necessidade de revelar o problema. Nestas circunstâncias, recomendam-se diferentes tentativas de sensibilizar o indivíduo a tomar a própria iniciativa; entre tanto, este deve ser informado da intenção e necessidade de quebra do acordo, como nos casos de HIV positivo, presença de gravidez próxima do termo e diferentes formas de violências. Nos casos omissos, onde o profissional detecte apenas os sinais e sintomas indiretos ou indicadores do problema, ressalta-se a responsabilidade profissional para buscar ajuda e apoio na denúnciae resolutividade do caso ${ }^{14,16}$.

$\mathrm{Na}$ abordagem do adolescente, a confidencialidade é reconhecida como uma das principais estratégias para a adesão às propostas de prevenção ou intervenção, pois incentiva a autonomiae responsabilização pelas próprias condutas e estilo de vida. Entretanto, é de fundamental importância que a família seja informada sobre os princípios da confidencialidade e a necessidade da mesma, tendo em vista mantê-los confiantes quanto à conduta profissional. Para ambos (pais/ responsáveis e adolescentes), é necessário informar sobrea possibilidade de rompimento daconfidencialidade, nos casos que indiquem risco de vida ou situações que necessitam da interferência da família. 0 profissional também deve estimular o adolescente a estabelecer o diálogo com seus familiares ${ }^{16,17}$.

\section{A resiliência e os fatores de risco e de proteção para a saúde}

Até 0 início da década de 80, as diferentes áreas das ciências médicas e sociais centraram suas investigações e aplicações práticas nas múltiplas "enfermidades", no intuito de descobrir as causas de problemas físicos e mentais. Entretanto, os modelos teóricos dessas investigações que procuravam explicar a alta probabilidade de resulta- dos negativos em função dos fatores de risco eram insuficientes para explicar os fenômenos da sobrevivência humana diante da adversida$\mathrm{de}^{5}$. Os indivíduos competentes, apesar das adversidades, eram denominados "invulneráveis", "fortes", "capazes de enfrentar", considerando-se que essa capacidade se tratava apenas deum atributo individual e permanente.

$\mathrm{Na}$ atualidade, esse fenômeno é denominado de "resiliência". O estudo da resiliência emergiu então dos estudos de risco, amplamente difundidos nos programas de saúde, os quais observaram que, em condições adversas, diante de um determinado evento não favorável, alguns indivíduos sobreviviam e desenvolviam competências para enfrentar e superar a adversidade. As pesquisas em resiliência originaram-se da área da psiquiatria e psicologia do desenvolvimento psicossocial da criança ${ }^{18,19}$.

A partir da década de 90, a maioria dos estudiosos passaram a enfocar a resiliência na perspectiva da abordagem ecossistêmica, considerando que o processo de adaptação é o resultado da dinâmica entre o ser humano e seu meio ambiente. Segundo esta abordagem, as trocas entre 0 indivíduo e o ambiente são as mais diferenciadas possíveis, uma vez queo indivíduo interage continuamente com o seu meio, sofrendo o impacto deste e, ao mesmo tempo, provocando reaçõe ${ }^{20}$.

0 termo "resiliência" tem sido utilizado pelas ciências sociais para caracterizar os indivíduos que se desenvolvem adequadamente num tempo dado e numa área psicossocial delimitada, apesar de conviver sob condiç̧ões adversas, em diferentes situações de risco, alcançando êxito social e adequação mental ${ }^{19,20}$.

No contexto ecossistêmico, resiliência consiste numa combinação de fatores pessoais e sociais, considerando a combinação de atributos pessoal, familiar, social, cultural que, através do tempo, permite ao indivíduo uma vida saudável, diante de situações adversas. A resiliência não éestática, é universal, nas diferentes comunidades, etnias, culturas, com características particulares, de acordo com o contexto em que se manifesta. Em diferentes momentos, o indivíduo pode ser vulnerável a uma determinada circunstância e resiliente à outra, a depender dos fatores de risco e de proteção existentes e das interações que se desenvolvem entre 0 indivíduo e 0 ambiente. Nenhum sujeito é completamente resiliente. A resiliência depende de diferentes circunstâncias, atuando num determinado momento, em um período de tempo e contexto sociocultural ${ }^{19,20,21}$. Importante contribuição para a abordagem 
voltada à promoção da resiliência, na prevenção de danos e problemas psicossociais, como delinqüência, drogadição, baixa escolaridade, desemprego, violência social e familiar, foi o conhecimento ea identificação dos fatores de risco, relacionados à vulnerabilidade em níveis individual, familiar, comunitário, assim como dos fatores protetores, que atuam protegendo ou neutralizando o efeito do agravo, mas, sobretudo, favorecendo o enfrentamento e superação da adversidade ${ }^{22,23}$.

No que diz respeito à infância e adolescência, as peculiaridades biopsicossociais relacionadas ao processo de crescimento, desenvolvimento pessoal (maturidade emocional e intelectual) e inserção social caracterizam este grupo como de alta vulnerabilidade aos agravos sociais envolvendo diferentes demandas que compreendem família, grupo social e os sistemas de atenção (pedagógico, saúde, assistência social, trabalho, lazer, esporte, outros) ${ }^{5}$.

Entre os riscos centrados na criança, destacam-se algumas diferenças: 1) pessoais - de gê nero (homens e mulheres podem apresentar diferentes respostas ao processo de adaptação social), - de temperamento (forte timidez, passividade ou alta reatividade), - de dificuldades cognitivas; 2 ) interpessoais - de agressividade interparental ou com os pares; 3) contextuais - de comunidades pobres ou pouco estimulantes. Esses fatores de riscos interagem entre si provocando e retroalimentando outras dificuldades, como rejeição dos pares e familiares, aumento da agressividade, atitudes violentas, fracasso acadêmico, isolamento, entre outras 22 .

Os fatores de origem familiar que contribuem para a inadaptação social mais destacados são a estrutura monoparental e o nível socioeconômico. Em relação à separação dos pais, sabe-se que é necessário distinguir entre os efeitos reais do evento e a história prévia do conflito que levou ao desfecho, sendo a qualidade das relações interparentais antes, durante e depois do divórcio o principal determinante da inadaptação. Os conflitos e as insatisfações conjugais estão associados a problemas exteriores e interiores à criança, podendo se manifestar como ansiedade, de pressão, cólera, somatizações. Outros fatores familiares apontados são as práticas educativas inadequadas, punitivas einstáveis, com encorajamento e reforço para comportamento anti-social 22,24 .

Os determinantes sociais, econômicos e culturais somam-se aos fatores familiares e individuais, favorecendo a perpetuação e a multiplicação de diferentes agravos. Freqüentemente, a po- breza está relacionada ao desemprego, pais solteiros, estresse parental, baixo nível educacional, assim como pobreza e conflitos maritais está relacionada à criminalidade e problemas de adaptação $0^{20}$.

Os fatores de risco e de proteção podem ser inatos ou adquiridos, internos ou externos, gerais ou específicos eagem modificando a resposta individual ou coletiva à adaptação social. U ma situação de estresse pode representar um agravo (fator de risco), assim como favorecer (fator de proteção) o desenvolvimento de habilidade e resistência para vencer e superar este agravo. Com efeito, as experiências exitosas atuam positivamente no desenvolvimento da criança; entretanto, 0 fator de proteção manifesta seus efeitos nas situações em que ocorre estresse, diante da possibilidade do agravo ou do acontecimento instalado.

Os fatores de risco e de proteção tendem a ser extensivos e cumulativos. 0 confronto com a adversidade em um contexto contribui para a adversidade em outras áreas. Do mesmo modo, um fator protetor pode contribuir para outras habilidades de enfrentar a adversidade. Crianças expostas a situações e experiências de traumas repetidos passam a sentir-se pouco saudáveis e incapazes. Ao contrário, o encorajamento desuas habilidades e o suporte para a autoconfiança fortalecem sua confiança e a manifestação da resiliência, ou seja, das atitudes positivas diante dos mesmos traumas ${ }^{23}$.

Os dois enfoques são complementares; enquanto 0 enfoque de risco prioriza a atuação no problema e características associadas ao dano (biológico ou social), sendo amplamente utilizado nos programas de atenção primária, o enfoque na resiliência atua no desenvolvimento das competências individuais e coletivas que protegem, preparando o indivíduo ou grupo para enfrentar e superar a adversidade (problema). Esssas estratégias de atuação devem estar pautadas nas normas culturais e sociais nas quais o sujeito se desenvolve.

Os programas e ações cujas abordagens estão baseadas na resiliência encontram-se fundamentados no conhecimento dos fatores de risco e de proteção existentes em determinado indivíduo, contexto e momento. Nestetipo de abordagem, a estratégia básica deve estar centrada no indivíduo e no grupo social, os quais se encontram expostos ao agravo, contribuindo para reforçar, viabilizar, desenvolver habilidades e competências individuais e coletivas que os permitam enfrentar e superar o evento, conseqüentemente, a qualidade de vida e de inserção e convívio social ${ }^{22}$.

A promoção da resiliência voltada à infância 
eadolescência alcança diferentes e múltiplasáreas, como as políticas sociais, assistência social, saúde, educação, trabal ho, justiça, entre outras, podendo atuar a nível individual, familiar e comunitário. Pode ser direcionada às distintas etnias e culturas, através da aplicação de programas e ações que possam reforçar as fortalezas, competências pessoais e coletivas, diante dos distintos fatores de vulnerabilidade e agravo.

As situações adversas, fatores de risco, assim como os mecanismos protetores, encontram-se presentes em todas as etapas da vida; portanto, a promoção da resiliência, assim como as intervenções, precisam considerar esses fatores preexistentes e convocar a participação individual e comunitária ${ }^{6,25}$.

Algumas competências desenvolvidas por crianças e adolescentes podem configurar-se como preditores de resiliência, podendo ser incrementadas através de programas voltados à proteção e promoção da qualidade de vida:

1. Capacidade de resolver problemas, capacidade de abstrair na busca de soluções novas para problemas cognitivos e sociais;

2. Competência social para comunicar-se, demonstrar empatia e resposta positiva ao contato, desde os primeiros anos de vida; as expressões "eu tenho, eu estou, eu posso" são atribuições verbais que representam competências;

3. Projeto de vida, proposta de futuro: relacionado ao sentido de autonomia, motivação para o futuro, sentido de anteci pação e coerência (preditores de resultados positivos, quanto à resiliência).

$O$ efeito protetor dos adultos contra os problemas desaúde de crianças e adolescentes

As investigações voltadas à infância e adolescência apontam que estes são agentes ativos do próprio desenvolvimento e, ao mesmo tempo, constatam o papel central dos adultos que fazem parte do meio ambiente, nos aspectos relacionados ao desenvolvimento físico, psicológico e social, quer seja pelos recursos à sua disposição ou à causa da responsabilidade social, que lhes cabe assegurar o pleno desenvolvimento.

$\mathrm{Na}$ atualidade, os conhecimentos sobre as necessidades da infância e juventude têm conduzido os pesquisadores e intervenantes (profissionais) à convicção cada vez maior de que, tanto no plano teórico, como prático, os problemas de saúde dessa população, atuais ou eventuais, são decorrentes da fragilidade, ausência e até mesmo da ruptura derelacionamentos com adultos significativos.

Pesquisas concluem que os adultos contribuem fortemente à saúde de suas crianças e adolescentes, como por exemplo: 1. favorecer a adoção de um comportamento de pronta atenção, coerente e estável, que contribui para o desenvolvimento físico mais harmonioso; 2 . estabelecer na criança um sentimento de confiança própria e nas outras pessoas; 3 . contribuir para que desenvolva competências necessárias para enfrentar a vida em sociedade, como a regulação das emoções ou da empatia. 0 conhecimento atual sobre o papel central dos adultos, como o pai e, principalmente, a mãe, aplica-se, sobretudo, para compreender melhor o desenvolvimento normal da criançą26,27.

Essas pesquisas têm tido dificuldade para explicar o fator protetor ou de resiliência do adulto, contra problemas de saúde, na presença deum contexto adverso. Como exemplo, num mesmo contexto de pobreza, qual seria o papel do adulto, diante de duas crianças, onde uma del as apresenta crescimento e desenvolvimento atrasado, enquanto outra se desenvolve normalmente, assim como um adolescente que pode apresentar comportamento delinqüente, enquanto outro enfrenta o mesmo ambiente adverso e não apresenta problemas de comportamento. Questiona-sequem seria mais importante: a natureza dos cuidados, a qualidade do acompanhamento, a qualidade dos model os parentais ou, preferencialmente, uma mel hor qualidade de relacionamento baseado na confiança?

Além destas questões, soma-se à problemática uma questão central que trata da constatação de que crianças e adolescentes em situação de vulnerabilidade precisam contar com adultos que não são seus pais. Freqüentemente, profissionais de serviço social, educadores, professores deparam-se com situações onde os pais estão desmotivados ou simplesmente ausentes. A questão é se esta relação de crianças ou adolescentes com adultos que não são os pais comporta as mesmas características quea relação parental, para resultar num efeito benéfico para a saúde destes?

A "Teoria do A pego" 28 tem sido comprovada e, ao curso dos últimos cinqüenta anos, tem caracterizado a função da relação pais-filhos, inspirando atualmente os protocolos de avaliação e de intervenção, os quais têm facilitado a integração social de crianças e adolescentes. A partir desta teoria, são ensinados aos estudantes e profissionais distinguir entre a qualidade das inte- 
rações e das relações, sabendo-se que esta última reflete a história nos primeiros anos de vida ( 0 a 2 anos - lactente). Precisamente, a organização das interações resultam na criação de esquemas relacionais - protótipos - que a criança ge neraliza com outras relações sociais (companheiros, amigos, colegas).

Experiências inovadoras baseadas na "Teoria do Apego" são realizadas através da estimulação das relações entre crianças e pais substitutos, educadores e outros, o que é pertinente nos casos de desmobilização dos pais biológicos. Por enquanto, a aplicação das experiências com crianças cuja saúde está em risco encontra-se ainda em fase de desenvolvimento, atendendo à consolidação de uma experiência "universidade - comunidade", a fim de assegurar a integridade da aplicação que diz respeito às pesquisas mais recentes.

Para realizar aplicações válidas com a noção de "relação" de adultos não parentes com as crianças, énecessário também considerar outras teorias e disciplinas. Por exemplo, muitos trabalhos em ethologia humana, em educação e psicologia se inclinaram sobre a noção de como ativar a re lação pai-filho; a relação educativa entre mestree aluno ou a aliança entre terapeuta e criança; tanto quanto as relações sem as quais as intervenções de promoção da saúde mental conduzem a criança a ganhos superficiais ${ }^{29}$. Entretanto, 0 forte potencial de aplicação destes domínios de pesquisa não encontrou ainda o caminho da total realização. Os contextos da ação de saúde pública com adultos e crianças na comunidade são habitualmentedistintos do contexto deum ambientecontrolado, da sala de atendimento, que permite ao profissional capitalizar sobre o vínculo desenvolvido com a criança, para ajudá-la a ter consciência dos comportamentos necessáriosà saúde. Sem renunciar a crer neste vínculo, o profissional deve muitas vezes olhar como a ação poderá ser realizada dentro de um contexto diversificado, que compreende o meio natural da criança, sua família, escola, comunidade.

Além do efeito protetor produzido pela qualidade da relação adulto-criança, baseado na confiança desenvolvida dentro da primeira infância, deve se ainda considerar outros processos de influência do adulto sobrea infância. Por exemplo, a qualidade de supervisão dos adultos, como a soma de comportamentos parentais que permitem ou proíbem. Estas atitudes são de fundamental importância para a aquisição de hábitos de vida saudável pela criança. Os estudiosos do desenvolvimento humano acreditam que 0 efeito máximo desse processo conhecido como "apren- dizagem social" se situa entre dois anos, atéa fase da entrada na escola. É possível então aos adultos estabelecer uma amostra de regras e hábitos de vida que a criança terá tendência a interiorizar, sob a condição de que seja feito por um adulto coerente e caloroso.

O papel do adulto como fator protetor ou de resiliência para a acriança depende da idade desta. Na primeira infância e fase préescolar, a imitação ${ }^{30}$, a aprendizagem social ${ }^{31}$ e 0 apego são processos importantes do efeito do adulto sobre a criança. $\mathrm{Na}$ adolescência, é verdadeiro e provável a influência de outros mecanismos dos adultos do convívio. Por exemplo, as estratégias de aprendizagem por abstração (ensinamentos de regras e valores de conduta social) baseadas em discussões interpessoais, democráticas e menos diretivas são mais acessíveis com os adolescentes, em relação às crianças ${ }^{32}$.

Um outro importante fenômeno psicológico entre adultos e crianças que facilita a adoção de comportamento (adaptado ou não) pela criança e adolescente é a qualidade dos model os ofertados pelos adultos. A tendência de imitação das crianças ( com piqueaos 2 anos mas durantetoda a vida, em diferentes graus) não deve ser negligenciada nas intervenções de saúde pública. Sem dúvida, o impacto da educação para a saúde realizada diretamente para a criança ou adolescente pode ser comprometido se os adultos ao seu redor não adotam comportamentos alvos.

Em resumo, sabe-se que é necessário incluir osadultosao redor das crianças eadolescentes nas estratégias de proteção e promoção da qualidade de vida. Estas estratégias devem ser aplicadas no estabelecimento deum vínculo deconfiança adulto-criança; na qualidade da supervisão e nos modelos dos adultos, para que a criança adote, ao final, comportamentos de vida saudável ${ }^{33}$.

\section{Redes de proteção e redes sociais}

A perspectiva de trabalho voltado à proteção $\mathrm{e}$ promoção da qualidade de vida para a infância e adolescência compreende uma dimensão integradora entre múltiplos setores, tanto nos aspectos macroestruturais (as políticas), como na articulação interna para mobilização das intervenções. A multiplicidade de mecanismos que interferem de forma positiva e/ou negativa para 0 adequado crescimento, desenvolvimento e integração social dessa população demanda a interação interinstitucional e interpessoal, reconhecida como trabalho em "rede". 
Existe consenso quanto à eficácia e operacionalidade da "rede de proteção", atuando entre múltiplas instâncias, com o objetivo comum de melhorar o impacto das propostas e ações, em atendimento às demandas. Essa estratégia detrabalho permitea descentralização quanto à tomada de decisões e concretização das ações. Diante dos diferentes níveis de complexidade e especificidades de cada setor (jurídico, educacional, saúde, assistência social, outros), essa "rede" possibilita a agregação de propostas e responde meIhor às necessidades, na garantia dos direitos e da atenção global a crianças e adolescentes, assim como na resolutividade dos problemas individuais e coletivos ${ }^{34}$.

Dessa forma, ao sefalar do trabal ho em "rede de proteção", compreende-se a noção básica de vinculação em torno de uma causa (fenômeno, evento), atuando de forma dinâmica, agindo e interagindo com esta atuação, construindo possibilidades de melhorias, quanto às condições necessárias ao desenvolvimento de crianças e adolescentes, principalmente aqueles que convivem com múltiplos fatores de risco nos macro (sistema social e econômico) e micro ambientes (família, escola, amigos, comunidade) ${ }^{34,35}$.

A prática de trabalho em "rede" voltado à infância e adolescência tem sido uma exigência da realidade social, nos países mais pobres, tendo em vista a necessi dade de mobilização em torno dos principais problemas sociais que podem comprometer a qualidade de vida, as perspectivas futuras e a integridade de milhares de crianças eadolescentes. A pobreza, a exclusão social, o tráfico de drogas, entre outros, são exemplos de problemas que atuam conjuntamente, retroalimentando danos, como a violência familiar esocial, assim como comprometendo a seguridade social e a esperança de vida.

No Brasil, a partir da década de 80 e com a ampla participação das organizações não governamentais - ONG, universidades, governo federal, estadual e municipal, associações de classe, associações comunitárias e comunidades, o trabalho em "rede", voltado à garantia de direitos, proteção e atendimento à infância eadolescência, vem sendo desenvolvido nas diferentes regiões, municípios e comunidades. Fazem parte do Sistema de Garantias de Direitos os Sistemas Judiciário, Administrativo de Atendimento e o de Políticas Setoriais, os quais devem encarregar-se de institucionalizar os Conselhos de Direitos Municipais e Estaduais, assim como os Conselhos Tutelares. No Poder Judiciário, atuam o Ministério Público, a Defensoria Pública, o Juizado, as Varas da infância e adolescência e as Delegacias especializadas ${ }^{35}$. $\mathrm{Na}$ área deAtendimento e de Políticas Setoriais, participam os Sistemas de Saúde, Educação, Trabalho, Assistência Social, Lazer, Esporte, Cultura, assim como o Sistema de D efesa ${ }^{34}$.

Para concluir, destaca-se ainda o papel das "redes de proteção" interinstitucional e transetorial, na formação e implementação das "redes sociais" que, ao contrário das primeiras, são informais, aparecem espontaneamente através da vinculação de pessoas e grupos de referência, possibilitando a partilha de valores culturais e posicionamentos comuns.

0 trabalho em "rede de proteção" viabiliza a formação das "redes sociais", estimulando o desenvolvimento de práticas integradas, a nível comunitário e contribuindo com a conscientização e mobilização social, voltada à proteção e cuidados com crianças e adolescentes, junto às famílias, lideranças comunitárias, amigos, parentes. A continuidade dessas práticas é apontada como um importante indicador do impacto positivo das propostas direcionadas à proteção, promoção eintervenção, no enfrentamento dos problemas psicossociais e de saúde ${ }^{35}$. 


\section{Referências}

1. Organização das Nações Unidas (ONU). Convenção Internacional de Direitos da Criança. Genebra: ONU; 1989.

2. Organization M ondiale de la Santé. Rapport M ondial sur la Violence et la Santé. Genéve: OM S; 2002.

3. Bezerra SC. Estatuto da Criança e do Adolescente: marco da proteção integral. In: Brasil. Ministério da Saúde. Violência faz mal à saúde. Brasília: M inistério da Saúde; 2004. p. 17-22.

4. M inayo M CS. Contextualização do debate sobre violência contra crianças e adolescentes. In: Brasil. M istério da Saúde. Violência faz mal à saúde. Brasília: M inistério da Saúde; 2004. p. 13-16.

5. Munist M, Santos H, Kotliarenco MA et al. Manual de identificación y promoción de la resiliencia en ninos y adolescentes. Washington: Organizacion Mundial de la Salud (OMS), Organizacion Pannamericana de la Salud (OPAS), Fundación Kellogg - Autoridad Sueca para el Desarrollo Internacional (ASDI); 1998.

6. Suárez Ojeda EN, Krauskopf D. El enfoque del riesgo y su aplicación a las conductas del adolescente: una perspectiva psico-social. Washington: OPS-OM S; 1995.

7. Carvalho RC, Pinheiro AM CM . Indicadores de saúde para a infância e adolescência. In: Costa COM , Souza $\mathrm{RP}$, organizadores. Semiologia eatenção primária à criança e adolescente. Rio de Janeiro: Revinter; 2005. p. 1-17.

8. Waiselfisz J. Relatório de desenvolvimento juvenil, 2003. Brasília: UNESCO; 2004.

9. Brasil. M inistério da Saúde. Indicadores e D ados Básicos para a Saúde (Rede Interagencial de Informações para Saúde - RIPSA). Brasília: M inistério da Saúde; 2003.

10. Brasil. Ministério da Saúde. Resolução 196/96 - Diretrizes enormas regulamentadoras de pesquisa envolvendo seres humanos. Brasília: M inistério da Saúde; 1996.

11. Taquette SR, Vilhena M M , Silva M M, Vale M P. Conflitos éticos no atendimento à saúde de crianças e adolescentes. Cad Saúde Pública 2005; 21 (6): 1717-1725.

12. Conselho Federal de Psicologia (CFP). Resolução 16/ 00 - Realização de pesquisa em psicologia com seres humanos. Brasília: CFP; 2000

13. Neiva-Silva L, Lisboa C, Koller SH. Bioética na pesquisa com crianças e adolescentes em situação de risco: dilemas sobre o consentimento e a confidencialidade. Jornal Brasileiro de D oenças Sexualmente Transmissíveis 2006; 17(3): 201-206.

14. Costa M CO, Costa PM, Oliveira Neto AF. Desafios da abordagem do adolescente: confidencialidadeeorientação contraceptiva. Jornal de Pediatria 1999; 74(1): 5-10.

15. Saito MI, Leal M M, Silva LEV. A confidencialidade no atendimento à saúde de adolescentes: princípios éticos. Pediatria de São Paulo 2000; 22(3): 1717-1725.

16. Sociedade de Pediatria de São Paulo. Aspectos éticos do atendimento médico do adolescente. Revista Paulista de Pediatria 1999; 17: 95-97.

17. Costa MCO, Souza RP. Abordagem da criança e do adolescente. In: Costa M CO, Souza RP, organizadores. Semiologia eatenção primária à criança eao adolescente. Rio de Janeiro: Revinter; 2005. p.76-91.
18. Garmezy N. Stress, competence and development. Continuities in the study of schizophrenic adults, children vulnerable to psychopathology and the search for stress-resistant children. American Journal of Orthopsychiatric 1987; 57(2):159-173.

19. Rutter $M$. La résilience: quelques considérations théoriques. In: Bolognini M, Plancherel B, Núñez R, Bettscchart R. Préadolescence théorie, recherche et clinique. Paris: ESF; 1994. p.147-158.

20. Waller MA. Resilience in ecosystemic context: evolution of the concept. American Journal of Orthopsychiatry 2001; 71(3):290-297.

21. Rutter M. Psychossocial resilience and protective mechanisms. American Journal of Orthopsychiatry 1987; 57(3):316-331.

22. Fortin L, Bigras $M$. La résilience des enfants: facteurs derisque, de protection et les modèles théoriques. Pratiques Psychologiques 2000; 1:49-63.

23. Fraser M W, Richman JM, Galinsky MJ. Risk, protection, and resilience: toward a conceptual framework for social work practice. Social Work Research 1999; 23(3):131-142.

24. Rutter M. Resilience in the face of adversity. Protective factors and resistance to psychiatric disorder. $\mathrm{Br}$ J Psychiatry 1985; 147: 598-611.

25. Boet S, Born M . Les configurations de risques comme approche de la résilience dans une étude longitudinale. RevueQ uébecoise de Psychologie 2001; 22(1):93-113.

26. Ainsworth M D. O bject relations, dependency, and attachment: a theoretical review of the infant-mother relationship. Child Development 1969; 40(4): 969-1025.

27. Zamberlan M AT. Interação mãe-criança: enfoques teóricos e implicações decorrentes de estudos empíricos. Estudos de Psicologia ( $\mathrm{N}$ atal) 2002; 7(2). p. 399406.

28. Bowlby J. Trilogia apego e perda. São Paulo: Martins Fontes; 1990.

29. McCabe R, PriebeS.The therapeutic relationship in the treatment of severe mental illness: a review of methods and findings. Int J Soc Psychiatr 2004; 50(2):115-128.

30. Prinz JJ. Imitation and moral development. Cambridge: MIT Press; 2005.

31. Kochanska G, Coy KC, Murray KT. The development of self-regulation in the first four years of life. Child D evelopment 2001; 72(4):1091-1111.

32. Baumrind D. Effective parenting during the early adolescent transition. In: Cowan PA, Hetherington EM editors. Family transitions. Hillsdale, NJ: Lawrence ErIbaum Associates Inc; 1991. p. 111-163.

33. Johnson G, Kent G, Leather J. Strengthening the parent-child relationship: a review of family interventions and their use in medical settings. Child: Care, $\mathrm{H}$ ealth and Development 2005; 31(1):25-32.

34. Deslandes S. Redes de proteção e redes sociais: uma práxis integradora. In: Brasil. M inistério da Saúde. Violência faz mal à saúde. Brasília: M inistério da Saúde; 2004. p. 135-141.

35. Oliveira VLA, Pfeiffer $L$, Ribeiro $C R$ et al. Redes de proteção: novo paradigma de atuação. In: Brasil. M inistério da Saúde. Violência faz mal à saúde. Brasília: Ministério da Saúde; 2004. p. 143-150. 